\title{
ON A JENSEN-TYPE INEQUALITY FOR $F$-CONVEX FUNCTIONS
}

\section{MirosŁAW ADAMEK}

Abstract. In this paper we present a counterpart of the Jensen inequality for $F$-convex functions. We present it in three forms: discrete, integral and operator. As we will see, the generality of the received results will allow to obtain, in particular cases, Jensen-type inequalities for strongly convex functions and superquadratic functions.

Mathematics subject classification (2010): 26D15, 26A51, 39B62.

Keywords and phrases: $F$-convex functions, support theorems, strongly convex functions, superquadratic functions, Jensen-type inequalities.

\section{REFERENCES}

[1] S. Abramovich, G. Jameson, G. Sinnamon, Refining Jensen's inequality, Bull. Sci. Math. Roum., (N.S.) 47 (95) (2004), 3-14.

[2] M. AdAMEK, On a problem connected with strongly convex functions, Math. Inequal. Appl. 19, 4 (2016), 1287-1293.

[3] A. Azócar, J. Gimenez, K. Nikodem, J. L. Sánchez, On strongly midconvex functions, Opuscula Math. 31, 1 (2011), 15-26.

[4] M. KlaRiČIĆ BAKUla, K. NiKODEM, On the converse Jensen inequality for strongly convex functions, J. Math. Anal. Appl.434, 1 (2016), 516-522.

[5] M. Kuczma, An Introduction to the Theory of Functional Equations and Inequalities. Cauchy's Equation and Jensen's Inequality, PWN-Uniwersytet Śląski, Warszawa-Katowice-Kraków, 1985, 2nd Edition: Birkhäuser, Basel-Boston-Berlin, 2009.

[6] N. Merentes, K. Nikodem, Remarks on strongly convex functions, Aequat. Math. 80 (2010), 193 199.

[7] K. Nikodem, Zs. PÁLes, Characterizations of inner product spaces by strongly convex functions, Banach J. Math. Anal. 5 (2011), no. 1, 83-87.

[8] C.P. Niculescu, L.E. Persson, Convex Functions and Their Applications. A Contemporary Approach, Second Edition, CMS Bokks in Mathematics, Springer, 2018, 415+xvii pp

[9] B.T. POLYAK, Existence theorems and convergence of minimizing sequences in extremum problems with restrictions, Soviet Math. Dokl. 7 (1966), 72-75.

[10] A.W. Roberts, D.E. Varberg, Convex Functions, Academic Press, New York-London, 1973. 